\title{
Restarted Simpler GMRES Augmented with Harmonic Ritz Vectors
}

\author{
Ravindra Boojhawon and Muddun Bhuruth \\ Department of Mathematics, University of Mauritius \\ Reduit, Mauritius \\ $\{r$.boojhawon, mbhuruth\}@uom.ac.mu
}

\begin{abstract}
We describe a method for improving the convergence of the Simpler GMRES method for problems with small eigenvalues. We augment the Krylov subspace with harmonic Ritz vectors corresponding to the smallest harmonic Ritz values. The advantage over augmented GMRES is that the problem of finding the minimal residual solution reduces to an upper triangular least-squares problem instead of an upperHessenberg least-squares problem. A second advantage is that harmonic Ritz pairs can be cheaply computed. Numerical tests indicate that augmented Simpler GMRES $(m)$ is superior to $\operatorname{Simpler} \operatorname{GMRES}(m)$ and requires a lesser amount of work than augmented $\operatorname{GMRES}(m)$.
\end{abstract}

\section{Introduction}

Restarting the GMRES method after each cycle of $m$ iterations slows down the convergence of the iterative method as each restart discards the information on the smallest eigenvalues which compose the solution. There are basically two approaches to reduce the negative effects of a restart: a preconditioning approach [1] and an augmented subspace approach [3]. These two approaches have been shown to improve the convergence of GMRES for problems with small eigenvalues. A cheaper implementation of GMRES can be achieved via the Simpler GMRES algorithm of Walker and Zhou [5]. The advantage is that the problem for finding the minimal residual solution reduces to an upper triangular least-squares problem instead of an upper-Hessenberg least-squares problem for GMRES. We show that for problems with small eigenvalues, it is possible to improve the convergence of restarted Simpler GMRES $(m)$ by augmenting the Krylov subspace with harmonic Ritz vectors corresponding to the smallest harmonic Ritz values which are the roots of the GMRES polynomial.

\section{Augmented Simpler GMRES}

Consider the nonsymmetric linear system

$$
A x=b,
$$

P.M.A. Sloot et al. (Eds.): ICCS 2002, LNCS 2330, pp. 393-402, 2002.

(C) Springer-Verlag Berlin Heidelberg 2002 
where $A \in \mathbb{R}^{n \times n}$ is nonsingular and $x, b \in \mathbb{R}^{n}$. Let $x_{0}$ be an initial guess such that $r_{0}=b-A x_{0} \neq 0$. Suppose that we have applied the Arnoldi process with $w_{1}=A r_{0} /\left\|A r_{0}\right\|_{2}$ to produce the orthonormal set of vectors $\left\{w_{1}, w_{2}, \ldots, w_{p-1}\right\}$ for some $p>1$. Let $\mathcal{K}_{p}(w)$ denote the Krylov subspace

$$
\mathcal{K}_{p}(w) \equiv \operatorname{span}\left\{w, A w, \ldots, A^{p-1} w\right\}
$$

If the GMRES iterate $x_{p-1}$ is not a solution of $(1)$, then $\mathcal{K}_{p}\left(w_{1}\right)=A\left(\mathcal{K}_{p}\left(r_{0}\right)\right)$ has dimension $p$ [5]. Suppose we want to augment the Krylov subspace with $k$ harmonic Ritz vectors $u_{1}, \ldots, u_{k}$.

Denote $m=p+k$ and let $Y_{m}$ be the $n \times m$ matrix whose last $k$ vectors are the harmonic Ritz vectors $u_{i}$ for $i=1, \ldots, k$. Also let $W_{m}$ to be the $n \times m$ matrix whose first $p$ columns are the vectors $w_{1}, \ldots, w_{p}$ and whose last $k$ columns are formed by orthogonalizing the vectors $\widetilde{u}_{i}=A u_{i}$, for $i=1, \ldots, k$ against the previous columns of $W_{m}$ by the following algorithm:

\section{AugmentedSArnoldi}

1. Start: Input $k$ approximate eigenvectors $U_{k}, \widetilde{U}_{k}=A U_{k}, m$ and $k$. Let $p=$ $m-k ; w_{1}=A r_{0} ; R_{1,1}=\left\|w_{1}\right\|_{2} ; w_{1}=w_{1} / R_{1,1} ;$

2. Iterate: For $i=2: m$

If $i \leq p, w_{i}=A w_{i-1}$; else, $w_{i}=\widetilde{u}_{i-p}$;

For $j=1, \ldots, i-1, R_{j, i}=w_{i}^{\mathrm{T}} w_{j} ; w_{i}=w_{i}-R_{j, i} w_{j}$;

$R_{i, i}=\left\|w_{i}\right\|_{2} ; w_{i}=w_{i} / R_{i, i}$

3. Output: If $k=0, Y_{m}=\left[\begin{array}{lll}r_{0} & W_{m-1}\end{array}\right]$; else, $Y_{m}=\left[\begin{array}{lll}r_{0} & W_{p-1} & U_{k}\end{array}\right]$.

The result of the above algorithm is the decomposition

$$
A Y_{m}=W_{m} R_{m}
$$

where $R_{m}$ is an $m \times m$ upper triangular matrix. If $k=0, Y_{m}=\left[\begin{array}{ll}r_{0} & W_{m-1}\end{array}\right]$ else $Y_{m}=\left[\begin{array}{lll}r_{0} & W_{p-1} & U_{k}\end{array}\right]$ where $U_{k}$ is a matrix whose $k$ columns are the $k$ harmonic Ritz vectors.

Writing the restarted problem in the form $A\left(\widetilde{x}-x_{0}\right)=r_{0}$, we find that the residual vector is given by

$$
r_{m}=r_{0}-W_{m} \widetilde{w}
$$

where $\widetilde{w}=R_{m} \widehat{y}=\left(\xi_{1}, \xi_{2}, \ldots, \xi_{m}\right)^{\mathrm{T}}$. From (4) and by the minimal residual criterion [2], we have $W_{m}^{\mathrm{T}} r_{m}=0$ and thus $r_{m}=r_{m-1}-\xi_{m} w_{m}$. The residual norm can be easily updated using

$$
\left.\left\|r_{m}\right\|_{2}=\sqrt{(}\left\|r_{m-1}\right\|_{2}^{2}-\xi_{m}^{2}\right)=\left\|r_{m-1}\right\|_{2} \sin \left(\cos ^{-1}\left(\xi_{m} /\left\|r_{m-1}\right\|_{2}\right)\right)
$$

The approximate solution $\widetilde{x}-x_{0}$ is a combination of the columns of $Y_{m}$ such that $\widetilde{x}-x_{0}=Y_{m} \widehat{y}=z_{m}$ and $\widehat{y}=R_{m}^{-1} \widetilde{w}=\left(\eta_{1}, \eta_{2}, \ldots, \eta_{m}\right)^{\mathrm{T}}$. It then follows 
that the augmented Simpler $\operatorname{GMRES}(m)$ correction is given by $z_{m}=Y_{m} \widehat{y}$ where

$$
z_{i}= \begin{cases}r_{0} \widehat{y}_{1}, & \text { if } i=1, \\ \left(r_{0}, w_{1}, w_{2}, \ldots, w_{i-1}\right) \widehat{y}_{i}, & \text { if } 1<i \leq p \\ \left(r_{0}, w_{1}, w_{2}, \ldots, w_{p-1}, u_{1}, \ldots, u_{i-p}\right) \widehat{y}_{i}, & \text { if } p<i \leq m\end{cases}
$$

In the Gram-Schmidt implementation of the augmented Simpler GMRES $(m)$ algorithm, the correction $z_{i}$ can be rewritten as

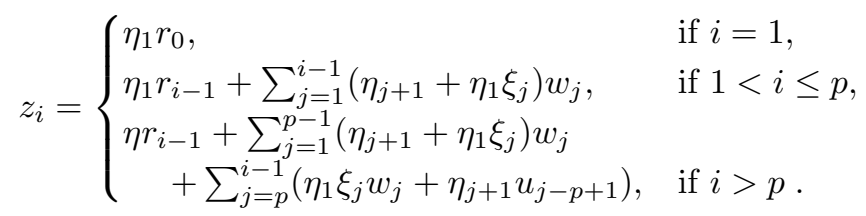

Harmonic Ritz vectors are computed by applying the minimal residual criterion. This criterion for finding an approximate eigenvector $u=Y_{m} \widetilde{g}_{m}$ of $A$ in $A \mathcal{K}_{m}\left(r_{0}\right)$ can be written as $[2]$

$$
(A u-\lambda u) \perp A \mathcal{K}_{m}\left(r_{0}\right) \Leftrightarrow W_{m}^{\mathrm{T}}\left(A Y_{m} \widetilde{g}_{m}-\lambda Y_{m} \widetilde{g}_{m}\right)=0
$$

Using (3), we have

$$
R_{m} \widetilde{g}_{m}=\lambda W_{m}^{\mathrm{T}} Y_{m} \widetilde{g}_{m}
$$

Denoting $\left(W_{m}^{\mathrm{T}} Y_{m}\right)^{-1}$ by $T_{m},(9)$ becomes

$$
T_{m} R_{m} \widetilde{g}_{m}=\lambda \widetilde{g}_{m}
$$

Noting that $T_{m}\left(R_{m} T_{m}\right) T_{m}^{-1}=T_{m} R_{m}$, the projected eigenvalue problem (9) becomes

$$
R_{m} T_{m} g_{m}=\widetilde{R}_{m} g_{m}=\lambda g_{m}
$$

and the approximate eigenvector becomes $u=Y_{m} \widetilde{g}_{m}=Y_{m}\left(T_{m} g_{m}\right)$.

If $k=0, Y_{m}=\left[r_{0} W_{m-1}\right]$, and thus $T_{m}$ and $\widetilde{R}_{m}$ can be easily obtained as shown in [2]. Now if $k>0$, then $Y_{m}=\left[r_{0} W_{p-1} U_{k}\right]$ and

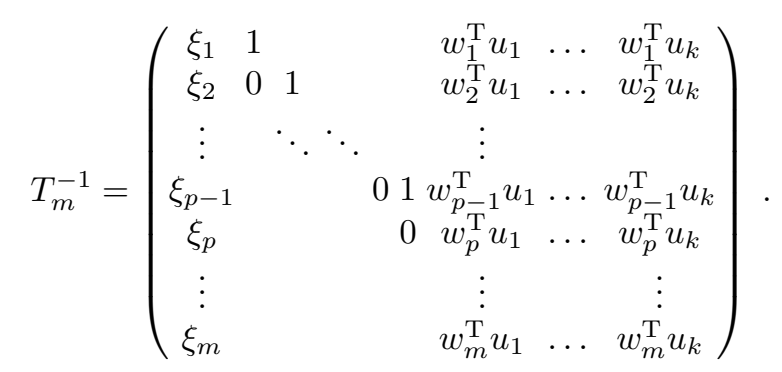


We then find that the matrix $T_{m}$ has the following structure:

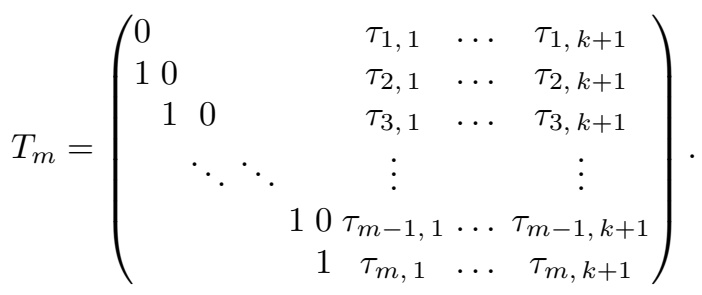

It is thus seen that the matrix $\widetilde{R}_{m}=R_{m} T_{m}$ is an upper Hessenberg matrix whose entries can be cheaply computed as follows: $\widetilde{R}_{i, j}=\sum_{q=i}^{q=m} R_{i, q} \tau_{q, j-p+1}$, for $i=1, \ldots, m, j=p, \ldots, m$ and $\widetilde{R}_{i, j}=R_{i, j+1}$, for $j=1, \ldots, p-1$, $i=1, \ldots, j+1$.

In the following, we describe the Modified Gram-Schmidt implementation of the augmented Simpler GMRES algorithm:

\section{SGMRESE}

1. Start: Choose $m$, maximum size of the subspace, and $k$, the desired number of approximate eigenvectors. Let $\epsilon$ be the convergence stopping criteria. Let $x_{0}=0$ such that $r_{0}=b, \rho_{0}=\left\|r_{0}\right\|_{2}, \rho=1, p=m-k$ and $w_{1}=A r_{0} /\left\|A r_{0}\right\|_{2}$, $r_{0}=r_{0} / \rho_{0}$.

2. First cycle: Apply the standard Simpler GMRES to produce $Y_{m}, W_{m}, R_{m}$, $r_{m}, x_{m}, \widetilde{w}$. Let $r_{0}=r_{m}$ and $x_{0}=x_{m}$. If $\left\|r_{m}\right\|_{2}$ is satisfied then stop, else $r_{0}=r_{0} /\left\|r_{0}\right\|$.

3. Form approximate eigenvectors: Compute $T_{m}=\left(W_{m}^{T} Y_{m}\right)^{-1}$ and $\widetilde{R}_{m}=$ $R_{m} T_{m}$ in order to solve $\widetilde{R}_{m} g_{j}=\lambda_{j} g_{j}$, for appropriate $g_{j}$. Form $u_{j}=$ $Y_{m}\left(T_{m} g_{j}\right)$ and $\widetilde{u}_{j}=W_{m}\left(\widetilde{R}_{m} g_{j}\right)$ for $j=1: k$.

4. Other cycles: Apply AugmentedSArnoldi with $\widetilde{U}_{k}, U_{k}, r_{0}, A, k$ and $m$ as inputs to generate $Y_{m}, W_{m}, R_{m}$. Find $\widetilde{w}=\left[\xi_{1}, \xi_{2}, \ldots, \xi_{m}\right]^{T}$ and $r_{m}=$ $r_{0}-W_{m} \widetilde{w}$ during the Simpler Arnoldi orthogonalization, that is, at each $i^{\text {th }}$ orthogonalization step we find $\xi_{i}=w_{i}^{T} r_{i-1}$ and $r_{i}=r_{i-1}-\xi_{i} w_{i}$. We also update $\rho$ using $\rho=\rho \sin \left(\cos ^{-1}\left(\xi_{i}\right) / \rho\right)$ and if $\rho \rho_{0} \leq \epsilon$ we go to $\mathbf{5}$.

5. Form the approximate solution: $\widehat{y}=R_{m}^{-1} \widetilde{w}$ and $x_{m}=x_{0}+\rho_{0} Y_{m} \widehat{y}=x_{0}+\rho_{0} z_{m}$ where $z_{m}$ is given by (7).

6. Restart: If $\left\|r_{m}\right\|_{2} \leq \epsilon$ is satisfied then stop, else $x_{0}=x_{m}, \rho_{0}=\left\|r_{m}\right\|_{2}$ and $r_{0}=r_{m} / \rho_{0}$. Set $\rho=1$ and go to 3 .

\subsection{Implementation Using Householder Reflectors}

In order to maintain orthogonality of the vectors to a high accuracy, it is preferable to use Householder orthogonalizations. Let $Q_{j}$ denote the Householder reflector that transforms only the $j^{\text {th }}$ through $n^{\text {th }}$ entries of the vectors on which 
it acts. Suppose $Q_{1}, Q_{2}, \ldots, Q_{i}$ are such that

$$
\left(\begin{array}{c}
R_{i} \\
0
\end{array}\right)= \begin{cases}Q_{i} A r_{0}, & \text { if } i=1, \\
Q_{i} \cdots Q_{1} A\left(r_{0}, w_{1}, \ldots, w_{i-1}\right), & \text { if } 1<i \leq p, \\
Q_{i} \cdots Q_{1} A\left(r_{0}, w_{1}, \ldots, w_{p-1}, u_{1}, \ldots, u_{i-p}\right), & \text { if } p<i \leq m .\end{cases}
$$

Since $R_{i}$ is invertible and

$$
\begin{cases}A r_{0}=w_{1} R_{1}, & \text { if } i=1, \\ A\left(r_{0}, w_{1}, \ldots, w_{i-1}\right)=\left(w_{1}, \ldots, w_{i}\right) R_{i}, & \text { if } 1<i \leq p, \\ A\left(r_{0}, w_{1}, \ldots, w_{p-1}, u_{1}, \ldots, u_{i-p}\right)=\left(w_{1}, \ldots, w_{i}\right) R_{i}, & \text { if } p<i \leq m .\end{cases}
$$

Then,

$$
Q_{i} \cdots Q_{1}\left(w_{1}, \ldots, w_{i}\right)=\left(\begin{array}{c}
I_{i} \\
0
\end{array}\right), \quad I_{i} \in \mathbb{R}^{i \times i} .
$$

and $w_{j}=Q_{1} \cdots Q_{i} e_{j}=Q_{1} \cdots Q_{j} e_{j}$ for $j=1, \ldots, i$ where $e_{j}$ is the $j^{\text {th }}$ column of identity matrix of order $n$. Letting $\widehat{y}_{i}=\left(\eta_{1}, \ldots, \eta_{i}\right)^{\mathrm{T}}$, and using (6) and (16), we have

$$
z_{i}= \begin{cases}Q_{1}\left(\eta_{1} Q_{1} r_{0}\right), & \text { if } i=1, \\ Q_{1} \cdots Q_{i}\left[\eta_{1} r_{i}+\left(\eta_{2}, \ldots, \eta_{i}, 0, \ldots\right)^{\mathrm{T}}\right], & \text { if } 1<i \leq p, \\ Q_{1} \cdots Q_{i}\left[\eta_{1} r_{i}+\left(\eta_{2}, \ldots, \eta_{p}, 0, \ldots\right)^{\mathrm{T}}\right]+\sum_{j=1}^{i-p} \eta_{p+j} u_{j}, & \text { if } p<i \leq m .\end{cases}
$$

Since $\left(Q_{i} \cdots Q_{1} r_{i}\right)^{T} e_{j}=\left(Q_{i} \cdots Q_{1} r_{i}\right)^{T}\left(Q_{i} \cdots Q_{1} w_{j}\right)=r_{i}^{\mathrm{T}} w_{j}=0$ for $j=1, \ldots, i$, it follows from (4) and (16) that $\xi_{i}=e_{i}^{\mathrm{T}} Q_{i} \cdots Q_{1} r_{0}$, and

$$
r_{i}=Q_{1} \cdots Q_{i}\left(\begin{array}{cc}
0 & \\
I_{n-i}
\end{array}\right) r_{i-1}, \quad I_{n-i} \in \mathbb{R}^{(n-i) \times(n-i)} .
$$

The following algorithm describes orthogonalization using Householder transformations.

\section{AugmentedSHArnoldi}

1. Start: Input $U_{k}, k$ approximate eigenvectors, $\widetilde{U}_{k}=A U_{k}, m$ and $k$. Let $p=m-k ; w_{1}=A r_{0} ;$

Find $Q_{1}$ such that $Q_{1} w_{1}=\left[R_{1,1}, 0, \ldots, 0\right]^{T}$;

$w_{1}=Q_{1} e_{1}$

2. Iterate: For $i=2: m$

If $i \leq p, w_{i}=Q_{i-1} \cdots Q_{1}\left(A w_{i-1}\right)$;

else, $w_{i}=Q_{i-1} \cdots Q_{1} \tilde{u}_{i-p}$;

Find $Q_{i}$ such that $Q_{i} w_{i}=\left[R_{1, i}, \ldots, R_{i, i}, 0, \ldots, 0\right]^{\mathrm{T}}$;

$w_{i}=Q_{1} \cdots Q_{i} e_{i}$

3. Output: If $k=0, Y_{m}=\left[\begin{array}{lll}r_{0} & W_{m-1}\end{array}\right]$; else, $Y_{m}=\left[\begin{array}{lll}r_{0} & W_{p-1} & U\end{array}\right]$. 
For the augmented Simpler GMRES algorithm using Householder reflections, we give below the steps that are different from the Gram-Schmidt version of the algorithm.

\section{SHGMRESE}

2. First cycle: Apply standard Simpler Householder GMRES.

4. Other cycles: Apply AugmentedSHArnoldi with $\widetilde{U}_{k}, U_{k}, r_{0}, A, k$ and $m$ as inputs to generate $Y_{m}, W_{m}, R_{m}$. Find $r_{i}=Q_{i} r_{i}$ and $\xi_{i}=e_{i}^{T} r_{i}$ at each $i^{\text {th }}$ orthogonalization step. We also update $\rho$ using $\rho=\rho \sin \left(\cos ^{-1}\left(\xi_{i}\right) / \rho\right)$ and if $\rho \rho_{0} \leq \epsilon$, we go to $\mathbf{5}$.

5. Form the approximate solution: $\hat{y}=R_{m}^{-1} \widetilde{w}$ and $\widetilde{x}_{m}=x_{0}+\rho_{0} Y_{m} \widehat{y}=x_{0}+\rho_{0} z_{m}$ where $z_{m}$ is given by (17).

\section{$3 \quad$ Numerical Experiments}

We describe the results of numerical experiments using Matlab. We compare restarted Simpler GMRES, SGMRES $(m)$ with the two augmented versions of the algorithm, namely, $\operatorname{SGMRESE}(p, k)$, the implementation using Gram-Schmidt orthogonalization and $\operatorname{SHGMRESE}(p, k)$, the Householder version. We also give the results for GMRES augmented with eigenvectors, $\operatorname{GMRESE}(p, k)[3]$. For each method, we compare the relative residual norm against the number of matrix-vector products (matvecs) and amount of work required (flops). We use $p=21$ Krylov vectors and $k=4$ harmonic Ritz vectors, and therefore $m=25$. In all experiments, the initial guess is the zero vector.

Experiment 1. We consider the first four test problems considered by Morgan [3]. The matrices are bidiagonal with 0.1 in each superdiagonal position. In Problem 1 , the matrix has entries $1,2,3, \ldots, 999,1000$ on the main diagonal. For Problem 2, the diagonal entries are 0.01, 0.02, 0.03, 0.04, 10, 11, . , 1004, 1005, for Problem 3, we have $-2,-1,1,2, \ldots, 997,998$ on the main diagonal and for Problem 4, the diagonal entries are 1, 1.01, 1.02, 1.03, 1.04, 2, 3, 4, . . , 995, 996. The right-hand side vector for each problem is a vector of ones.

We show in Fig. 1 the convergence history for Problem 2. This problem is difficult because of the small eigenvalues $0.01,0.02,0.03,0.04$. In this case, SGMRES(25) stagnates because the Krylov subspace is not large enough to develop approximations to the small eigenvalues. For roughly the same amount of work, SGMRES reaches a relative residual norm of 2.02E-2 whereas SGMRESE reaches a norm of $8.95 \mathrm{E}-11$. On the other hand, we observe that the three augmented methods are convergent, each reaching a relative residual norm of 2.8E-5 after 207 matvecs. However, the work required by SGMRESE, the Gram-Schmidt implementation, is $2.29 \mathrm{E} 7$ which is less than that required by GMRESE, approximately 3.48E7 flops. Also, SHGMRESE requires 6.12E7 flops to reach the same relative residual norm. 


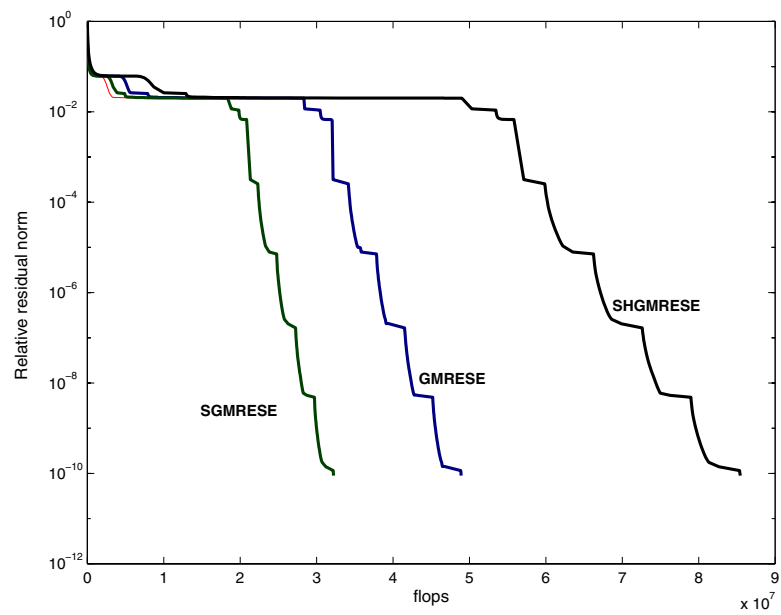

Fig. 1. Convergence history for example 2 of [3]

Table 1. Results for problems 1 to 4 of [3]

\begin{tabular}{|c|l|c|c|c|}
\hline Problem & Method & matvecs & $\left\|r_{i}\right\|_{2} /\left\|r_{0}\right\|_{2}$ & flops \\
\hline \multirow{3}{*}{1} & SGMRES & 523 & $9.73 \mathrm{E}-11$ & $3.30 \mathrm{E} 7$ \\
& GMRESE & 239 & $9.38 \mathrm{E}-11$ & $4.28 \mathrm{E} 7$ \\
& SGMRESE & 239 & $9.38 \mathrm{E}-11$ & $2.73 \mathrm{E} 7$ \\
& SHGMRESE & 239 & $9.38 \mathrm{E}-11$ & $7.07 \mathrm{E} 7$ \\
\hline \multirow{3}{*}{2} & SGMRES & 500 & $2.02 \mathrm{E}-2$ & $3.17 \mathrm{E} 7$ \\
& GMRESE & 280 & $8.95 \mathrm{E}-11$ & $4.89 \mathrm{E} 7$ \\
& SGMRESE & 280 & $8.95 \mathrm{E}-11$ & $3.22 \mathrm{E} 7$ \\
& SHGMRESE & 280 & $8.95 \mathrm{E}-11$ & $8.54 \mathrm{E} 7$ \\
\hline \multirow{3}{*}{3} & SGMRES & 500 & $8.89 \mathrm{E}-3$ & $3.17 \mathrm{E} 7$ \\
& GMRESE & 399 & $9.64 \mathrm{E}-11$ & $6.92 \mathrm{E} 7$ \\
& SGMRESE & 399 & $9.64 \mathrm{E}-11$ & $4.55 \mathrm{E} 7$ \\
& SHGMRESE & 399 & $9.64 \mathrm{E}-11$ & $1.18 \mathrm{E} 8$ \\
\hline \multirow{6}{*}{4} & SGMRES & 511 & $9.86 \mathrm{E}-11$ & $3.40 \mathrm{E} 7$ \\
& GMRESE & 425 & $9.78 \mathrm{E}-11$ & $7.66 \mathrm{E} 7$ \\
& SGMRESE & 425 & $9.78 \mathrm{E}-11$ & $4.95 \mathrm{E} 7$ \\
& SHGMRESE & 425 & $9.78 \mathrm{E}-11$ & $1.32 \mathrm{E} 8$ \\
\hline
\end{tabular}

Table 1 shows the results for the four test problems. For Problem 1, we note that SGMRES requires 523 matrix-vector products and a work of 3.30E7 to reach a relative residual norm of $9.73 \mathrm{E}-11$ whereas the augmented algorithm SGMRESE needs 239 matrix-vector products and only $2.73 \mathrm{E} 7$ flops to reach a norm of 9.38E-11. Also observe that SGMRESE performs better than GMRESE. 
The Householder version of augmented SGMRES requires more work because of the higher orthogonalization costs. The matrix in Problem 3 is indefinite and the results show that after 500 matrix-vector products, the relative residual norm reached by SGMRES is 8.89E-3 whereas SGMRESE and GMRESE reach a residual norm of 9.64E-11 after 399 matrix-vector products. However, note that the work required by GMRESE is approximately $50 \%$ more than the work required by SGMRESE. As mentioned by Morgan [3], Problem 4 is designed to be difficult for augmented methods. The results seem to confirm this as the augmented version of Simpler GMRES is not better than SGMRES. We note that SGMRESE takes a greater amount of work than SGMRES to reach approximately the same relative residual norm of $9.8 \mathrm{E}-11$.

For Problem 3, we show in Table 2 the residual norm reached and the amount of flops required by SGMRESE and GMRESE after 5, 10, 15 and 20 runs. Note that a run is the iteration between two successive restarts. Both GMRESE and SGMRESE, require the same number of matrix-vector products for the different runs. However we remark that in each case, the amount of work required by SGMRESE is less than the work required by GMRESE to reach the same residual norm.

Table 2. Residual norm and flops after different runs for Problem 3

\begin{tabular}{|l|l|l|l|l|l|l|l|l|}
\hline & \multicolumn{2}{|c|}{$\begin{array}{c}\text { After } \\
5 \text { runs }\end{array}$} & \multicolumn{2}{c|}{$\begin{array}{c}\text { After } \\
10 \text { runs }\end{array}$} & \multicolumn{2}{c|}{$\begin{array}{c}\text { After } \\
15 \text { runs }\end{array}$} & \multicolumn{2}{c|}{$\begin{array}{c}\text { After } \\
20 \text { runs }\end{array}$} \\
\hline Method & $\left\|r_{i}\right\|_{2}$ & flops & $\left\|r_{i}\right\|_{2}$ & flops & $\left\|r_{i}\right\|_{2}$ & flops & $\left\|r_{i}\right\|_{2}$ & flops \\
\hline SGMRESE & 0.22 & $1.14 \mathrm{E} 7$ & $8.3 \mathrm{E}-5$ & $2.38 \mathrm{E} 7$ & $2.1 \mathrm{E}-8$ & $3.62 \mathrm{E} 7$ & $5.4 \mathrm{E}-12$ & $4.86 \mathrm{E} 7$ \\
GMRESE & 0.22 & $1.74 \mathrm{E} 7$ & $8.3 \mathrm{E}-5$ & $3.65 \mathrm{E} 7$ & $2.1 \mathrm{E}-8$ & $5.55 \mathrm{E} 7$ & $5.4 \mathrm{E}-12$ & $7.46 \mathrm{E} 7$ \\
\hline
\end{tabular}

Similar results for Problem 2 show that, after 12 runs, GMRESE requires approximately $4.32 \mathrm{E} 7$ flops to reach a residual norm of 1.69E-7, whereas SGMRESE requires $2.88 \mathrm{E} 7$ flops to reach the same norm.

Experiment 2. We consider matrices, SHERMAN4, SHERMAN1 and SAYLR3 from the Harwell-Boeing sparse matrix collection. SHERMAN1 is nonsymmetric of dimension 1000 and has 3750 nonzero entries, SHERMAN4 is nonsymmetric, has dimension 1104 and 3786 nonzero entries and SAYLR3 is a nonsymmetric matrix, has dimension 1000 and 375 nonzero entries. The convergence history for SHERMAN4 in Fig. 3 shows that SGMRESE performs better than the other methods. In Table 3, we show the number of matrix-vector products, the relative residual norm and the amount of work required for the three matrices. For SHERMAN1, we observe that Simpler GMRES requires 5032 matrix-vector products to reach a relative residual norm of $9.99 \mathrm{E}-11$ whereas the augmented version SGMRESE requires only 1304 matrix-vector products to reach approximately the same norm. Comparing the amount of work for the two methods, we 
observe that SGMRESE requires a lesser amount. For all the three test problems, we observe that SGMRESE performs better than GMRESE.

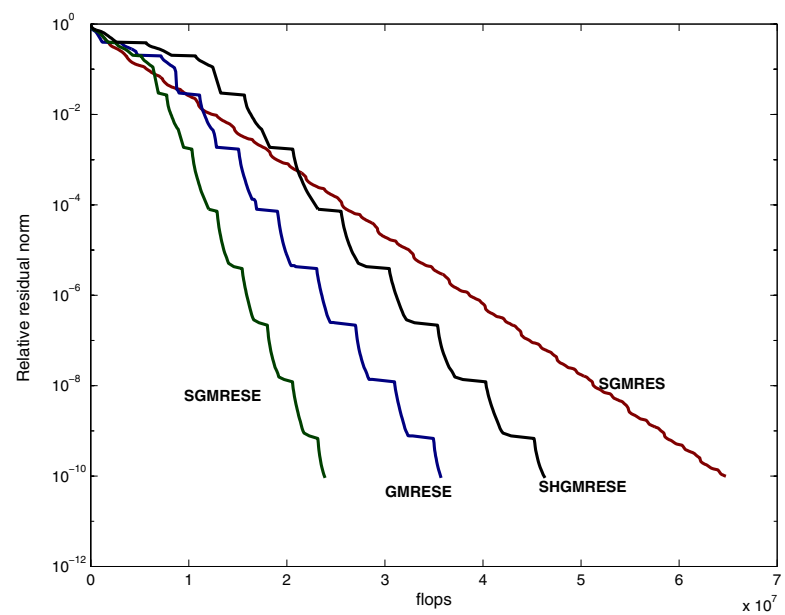

Fig. 2. Convergence for the SHERMAN4

Table 3. Results for Harwell-Boeing matrices

\begin{tabular}{|c|l|c|c|c|}
\hline Matrix & Method & matvecs & $\left\|r_{i}\right\|_{2} /\left\|r_{0}\right\|_{2}$ & flops \\
\hline \multirow{5}{*}{ SHERMAN4 } & SGMRES & 891 & $9.96 \mathrm{E}-11$ & $6.48 \mathrm{E} 7$ \\
& GMRESE & 208 & $9.11 \mathrm{E}-11$ & $3.57 \mathrm{E} 7$ \\
& SGMRESE & 208 & $9.11 \mathrm{E}-11$ & $2.39 \mathrm{E} 7$ \\
& SHGMRESE & 208 & $9.11 \mathrm{e}-11$ & $4.63 \mathrm{E} 7$ \\
\hline \multirow{5}{*}{ SHERMAN1 1} & SGMRES & 5032 & $9.99 \mathrm{E}-11$ & $3.36 \mathrm{E} 8$ \\
& GMRESE & 1304 & $9.90 \mathrm{E}-11$ & $2.26 \mathrm{E} 8$ \\
& SGMRESE & 1304 & $9.90 \mathrm{E}-11$ & $1.48 \mathrm{E} 8$ \\
& SHGMRESE & 1304 & $9.90 \mathrm{e}-11$ & $3.18 \mathrm{E} 8$ \\
\hline \multirow{5}{*}{ SAYLR3 } & SGMRES & 3951 & $9.99 \mathrm{E}-11$ & $2.64 \mathrm{E} 8$ \\
& GMRESE & 1142 & $9.98 \mathrm{E}-11$ & $2.06 \mathrm{E} 8$ \\
& SGMRESE & 1142 & $9.98 \mathrm{E}-11$ & $1.37 \mathrm{E} 8$ \\
& SHGMRESE & 1142 & $9.98 \mathrm{E}-11$ & $3.48 \mathrm{E} 8$ \\
\hline
\end{tabular}

We show in Table 4 the residual norm reached and the amount of work required after different runs for SHERMAN4. Observe that the amount of work required by augmented GMRES is roughly $40 \%$ more than the work required by augmented Simpler GMRES. 
Table 4. Residual norm and flops after different runs for SHERMAN4

\begin{tabular}{|c|c|c|c|c|c|}
\hline & $\begin{array}{l}\text { After } \\
5 \text { runs }\end{array}$ & & $\begin{array}{l}\text { fter } \\
\text { uns }\end{array}$ & $\begin{array}{c}\text { Af } \\
10\end{array}$ & $\begin{array}{l}\text { fter } \\
\text { runs }\end{array}$ \\
\hline Method & \begin{tabular}{|l|l|}
$r_{i} \|_{2}$ & flops \\
\end{tabular} & $\left\|r_{i}\right\|_{2}$ & flops & $\left\|r_{i}\right\|_{2}$ & flops \\
\hline$\overline{S G}$ & \begin{tabular}{|l|l|}
$4.2 \mathrm{E}-3$ & $1.21 \mathrm{E} 7$
\end{tabular} & $7.2 \mathrm{E}-7$ & $1.98 \mathrm{E} 7$ & $4.8 \mathrm{E}-9$ & $92.39 \mathrm{Et}$ \\
\hline GMRESE & \begin{tabular}{|l|l}
$4.2 \mathrm{E}-3$ & $1.69 \mathrm{E} 7$
\end{tabular} & $7.2 \mathrm{E}-7$ & $2.88 \mathrm{E} 7$ & $4.8 \mathrm{E}-9$ & $93.57 \mathrm{E} 7$ \\
\hline
\end{tabular}

\section{Conclusion}

We have described a method to improve the convergence of Simpler GMRES for problems with small eigenvalues. Numerical tests indicate that using GramSchmidt orthogonalizations to implement the algorithm, significant reduction in the amount of work required for convergence can be achieved over augmented GMRES. More numerical experiments using preconditioning techniques for other problems will be reported in another work.

\section{References}

1. Erhel, J., Burrage, K., Pohl, B.: Restarted GMRES preconditioned by deflation. J. Comput. Appl. Math. 69 (1996) 303-318

2. Goossens, S., Roose, D.: Ritz and harmonic Ritz values and the convergence of FOM and GMRES. Numer. Lin. Alg. Appl. 6 (1999) 281-293

3. Morgan, R.B.: A restarted GMRES method augmented with eigenvectors. SIAM J. Matrix Anal. Appl. 16 (1995) 1154-1171

4. Saad, Y., Schultz M.H.: GMRES: A generalized minimal residual algorithm for solving nonsymmetric linear systems. SIAM J. Sci. Statist. Comput. 7 (1986) 865869

5. Walker, H.F., Zhou L.: A Simpler GMRES. Numer. Lin. Alg. with Appl. 1 (1994) 571-581 